\title{
Game Theory in Signal Processing and Communications
}

\author{
Holger Boche (EURASIP Member), ${ }^{1}$ Zhu Han, $^{2}$ Erik G. Larsson, ${ }^{3}$ and Eduard A. Jorswieck ${ }^{4}$ \\ ${ }^{1}$ Department of Mobile Communications, School of Electrical Engineering and Computer Sciences, \\ Technical University of Berlin, Berlin, Germany \\ ${ }^{2}$ Wireless Networking, Signal Processing and Security Lab, Department of Electrical and Computer Engineering, \\ University of Houston, Houston, TX 77004, USA \\ ${ }^{3}$ Division of Communication Systems, Department of Electrical Engineering (ISY), Linköping University, SE-581 83 Linköping, Sweden \\ ${ }^{4}$ Communications Laboratory, Faculty of Electrical Engineering and Information Technology, Dresden University of Technology, \\ 01062 Dresden, Germany
}

Correspondence should be addressed to Eduard A. Jorswieck, jorswieck@ifn.et.tu-dresden.de

Received 12 November 2009; Accepted 16 November 2009

Copyright (C) 2009 Holger Boche et al. This is an open access article distributed under the Creative Commons Attribution License, which permits unrestricted use, distribution, and reproduction in any medium, provided the original work is properly cited.

Game theory is the study of the ways in which strategic interactions among rational players produce outcomes with respect to the preferences (or utilities) of those players. Games are represented in different forms, namely, the extensive form, normal form, and characteristic and partition function forms. Noncooperative games are usually defined by normal and extensive form games, whereas cooperative games are defined by characteristic and partition function form games. In noncooperative games, the players decide without communication and coordination on their strategies by selfishly maximizing their individual payoff functions. Equilibrium concepts characterize outcomes of noncooperative games. In cooperative games, players choose their strategies by negotiation and cooperation. In general the theory provides a structured approach to many important problems arising in signal processing and communications, notably resource allocation and distributed transceiver optimization. Recent applications also occur in other emerging fields such as cognitive radio, spectrum sharing, and multihop sensor and adhoc networks. Game theory is also used in cross-layers optimization where radio resource management on physical and MAC layers is connected with economic decisions on service and application layers.

This special issue presents research in applied game theory for signal processing and communications. In order to solve conflict situations in communication and in particular wireless networks, game theory provides a systematic approach. The limited resources in wireless communications are spectrum, space, power, and time. Game theory is used to analyze spectrum sharing, resource allocation, power control, transmit strategies, and network etiquette in wireless multiuser networks. The interest in these methods is increased significantly during the last ten years in the networking and signal processing communities.

In this special issue we, have been able to cellect seven papers on a variety of topics.

The first paper, "Saddle-point properties and nash equilibria for channel games" authored by Rudolf Mathar and Anke Schmeink studies a game between transmitter and nature represented by the channel. Equilibrium strategies are characterized for the noncooperative two-person zero-sum game in standard form.

Matthew Nokleby and A. Lee Swindlehurst analyze the cooperative bargaining on the MISO interference channel in the second paper entitled "Bargaining and the MISO interference channel". The optimal Kalai-Smorodinsky beamformers are derived. Joint scheduling, beamforming, and power control show significant performance gains for strong interference and scenarios with more links than transmit antennas.

The third paper, "Spectrum allocation for decentralized transmission strategies: properties of nash equilibria" authored by Peter von Wrycza et al. studies two links coexisting in the same area at the same time on the same frequency. The conflict is modeled as game in normal form and the Nash equilibrium is characterized. A modified utility function is proposed to improve the outcome in terms of sum rate efficiency. 
In the fourth paper, "Stackelberg contention games in multiuser networks", Jaeok Park and Mihaela van der Schaar propose to model a Stackelberg game for noncooperative communications in order to improve the efficiency of the equilibrium outcome. The network manager leads the game and decides on an intervention rule. The amount of additional information required is studied and it is shown that relaxing the requirements lead to small performance loss.

Zhu Han et al. in their paper "Auction-based resource allocation for cooperative video transmission protocols over wireless networks" propose wireless video transmission protocols for end-to-end distortion minimization. For multiuser networks, the share auction approach is extended into cooperative video transmission. Experimental results illustrate the gain in terms of peak signal to-noise ratio.

The sixth paper, Modeling Misbehavior in Cooperative Diversity: A Dynamic Game Approach by Sintayehu Dehnie and Nasir Memon, designs cooperative diversity protocols and analyzes misbehavior for the game in extensive form. Misbehavior turns out to be an evolutionary stable strategy. Therefore, a mechanism to detect and mitigate effects of misbehavior is developed based on a dynamic game formulation with incomplete information.

Ming-Hua Lin, Jung-Fa Tsai, and Yinyu Ye in their paper Budget Allocation in a Competitive Communication Spectrum Economy study a competitive communication spectrum market. A condition for existence of a competitive equilibrium with physical power demand requirements for the communication spectrum market with Shannon utility is derived. A centralized algorithm to reach a desired competitive equilibrium for satisfying power demands or balancing individual utilities is proposed.

We received an overwhelming response to the issue. We would like to thank all authors for their contributions to our issue, the reviewers for their help in selecting papers, and finally the editor Phillip Regalia for his support.

Holger Boche Zhu Han

Erik G. Larsson

Eduard A. Jorswieck 